\title{
Application of RFID Technology in Teaching Archive Management
}

\author{
Min $\mathrm{Hu}^{1, \mathrm{a}}$ and Wei Dong ${ }^{2, \mathrm{~b}}$ \\ ${ }^{1}$ School of Politics, Southwest University for Nationalities, Chengdu 610041, China; \\ ${ }^{2}$ School of Electrical and Information Engineering, Southwest University for Nationalities, Chengdu \\ 610041, China. \\ a skbhumin2006@sina.com, ${ }^{b}$ brant.dong@qq.com,
}

Keywords: RFID, RFID tag, barcodes, teaching archive.

\begin{abstract}
The college teaching archive (CTA) management faces a few problems: 1) no specialized storage rooms for teaching archives; 2) no professional for managing teaching archives, and 3) not being supported by database technology. In order to change this situation, the application of RFID technology is discussed, and an application scheme is presented for the purposes of promoting the automation of the college teaching archive management.
\end{abstract}

\section{Introduction}

Radio-frequency identification (RFID) is the wireless non-contact use of radio-frequency electromagnetic fields to transfer data, for the purposes of automatically identifying and tracking tags. Unlike a barcode, the tag does not necessarily need to be within line of sight of the reader, and may be embedded in the tracked object. RFID uses an electronic chip, usually applied to a substrate to form a label, that is affixed to a product, case, pallet or other package. The information it contains may be read, recorded, or rewritten. RFID can be used in a variety of applications, such as: access management, tracking of goods, tracking of persons and animals, toll collection and contactless payment [1]. In China, libraries such as National Library, Shengzheng Library, Wuhan Library, and Shantou University Library have applied RFID system, and the social and economic benefits are obtained. Today RFID door cards and RFID meal cards are common, and RFID ETC(Electronic Toll Collection) cards are more and more on the highway. However, RFID tags are not used in college teaching archive management.

The college teaching archives (CTA) are valuable teaching file materials which are produced during teaching management, teaching practice, and education research activities. They have types of text, chart, video, and disk, and play an important role in the college development. But the CTA management faces a few problems: 1) no specialized storage rooms for teaching archives; 2) no professional for managing teaching archives, at present as a part-time job taken by college secretaries; and 3) not being supported by database technology, the entity files and electronic files are managed manually, this causes problems for looking up or using the teaching archives. Therefore, the study on automatic management of college teaching archives possesses practical significance. For this reason, the application of RFID technology is discussed in the paper, and an application scheme of the CTA management system is presented.

\section{The Hardware Scheme of Teaching Archive Management System Based on RFID}

The hardware of college teaching archive management system includes RFID subsystem, RFID information processing system and the server of electronic archive database. The RFID subsystem consists of RFID tags, RFID readers and antennas.

RFID Tag Application. RFID tags can be either passive, active or battery-assisted passive. A passive RFID tag has no battery. An active tag has an on-board battery. A battery-assisted passive tag has a small battery on board and is activated when in the presence of an RFID reader. A passive tag is cheaper and smaller but read at short ranges. An active tag is operated at hundreds of meters but at 
higher cost. Since storage room area for college teaching archives is relatively small, and the passive RFID tags are matured, reliable, and cheaper, thus the passive RFID tags using Low to moderate frequency band is chosen in our scheme.

RFID Tag Information Set. According to the use of different purposes, RFID tags are divided into archive tags and location tags in the CTA management system. The information items stored in the archive tags include archive type, archive name, archive number, archive security class, storage time, and storage life. The information items stored in the location tags include location type, location number, stored archive number interval, number of stored archives.

RFID Tag Installation. The archive tags are packaged in the form of card, and glued on the top of the archive box. If a few archives are put side by side, then glued in turn on the top, middle, and bottom of the archive box in order to avoid interference among them when information is transmitted. The location tags are installed directly on the underside of the support beam. Since storage room for college teaching archives is relatively small, the requirement is satisfied if one location tag is stalled in every independent compartment of the archive cabinet.

RFID Reader. RFID reader is a device which can read tag information (sometimes can write tag information too), and can be classified into two types, the fixed readers and the hand-held readers. The fixed readers are mounted at the entrance of college teaching archive storage room, and the hand-held readers are used to collect information or to make an inventory of teaching archives in the CTA storage room. Since storage room area for college teaching archives is relatively small, it is simple, practical and economic to make an inventory of teaching archives in the CTA storage room by using the hand-held readers.

RFID Antennas. Passive, active and reader are the most common RFID tag antenna types. A passive RFID tag antenna is a basic type of antenna. It works by converting a magnetic field into a power source that the tag uses to communicate. The passive RFID tag antennas tend to be very cost effective and are typically disposable. The second type is an active RFID tag antenna. Instead of just transmitting a signal, an active antenna can also receive signals. Reader antennas are a third type. Instead of just operating on one radio frequency, these antennas have the capability of communicating on several at once. They can send and receive data just like an active antenna [2]. Since storage room area for college teaching archives is relatively small, a reader antenna is mounted at its entrance for inquiring about the information stored in the teaching archive tags which are taken out from the storage room.

The Choice of Backstage Database Server. MySQL is the world's most popular open source database software. With its superior speed, reliability, and ease of use, it is a popular choice of database for use in web applications. MySQL uses the standard language SQL for accessing databases, it has five editions, the Enterprise Edition, the Standard Edition, the Classic Edition, the Cluster CGE, and the Community Edition [3]. In comparison with Oracle、DB2、SQL Server, MySQL has its weakness, but the functions it has provided are more than sufficient for the CTA management system. Since MySQL is open source software, the running cost can be reduced. Hence, MySQL database server is chosen as backstage database server for the CTA management system.

\section{The Software Scheme of Teaching Archive Management System Based on RFID}

The college teaching archive management system based on RFID is shown in Fig. 1, which consists of electronic archive database, RFID information processing system, RFID subsystem, archive query subsystem, archive destruction subsystem, archive location subsystem, and monitoring alarm subsystem. The electronic archive database is run on the backstage server, and electronic archive files are managed through the database. The RFID subsystem accomplishes reading and writing the CTA information of entity teaching archives, and the obtained information from the RFID subsystem is processed and stored in the database by RFID information processing system. Clearly, the electronic archive files are interrelated with entity archive files via the RFID subsystem and RFID information processing system. Only after authorization of RFID information processing system, the archive query subsystem, archive destruction subsystem and archive location subsystem can 
accomplish operating the electronic archive files and entity archive files. When the entity archive files without checkout are taken out from the storage room, RFID information processing system will monitor this case through the RFID subsystem, and let the monitoring alarm subsystem give an alarm.

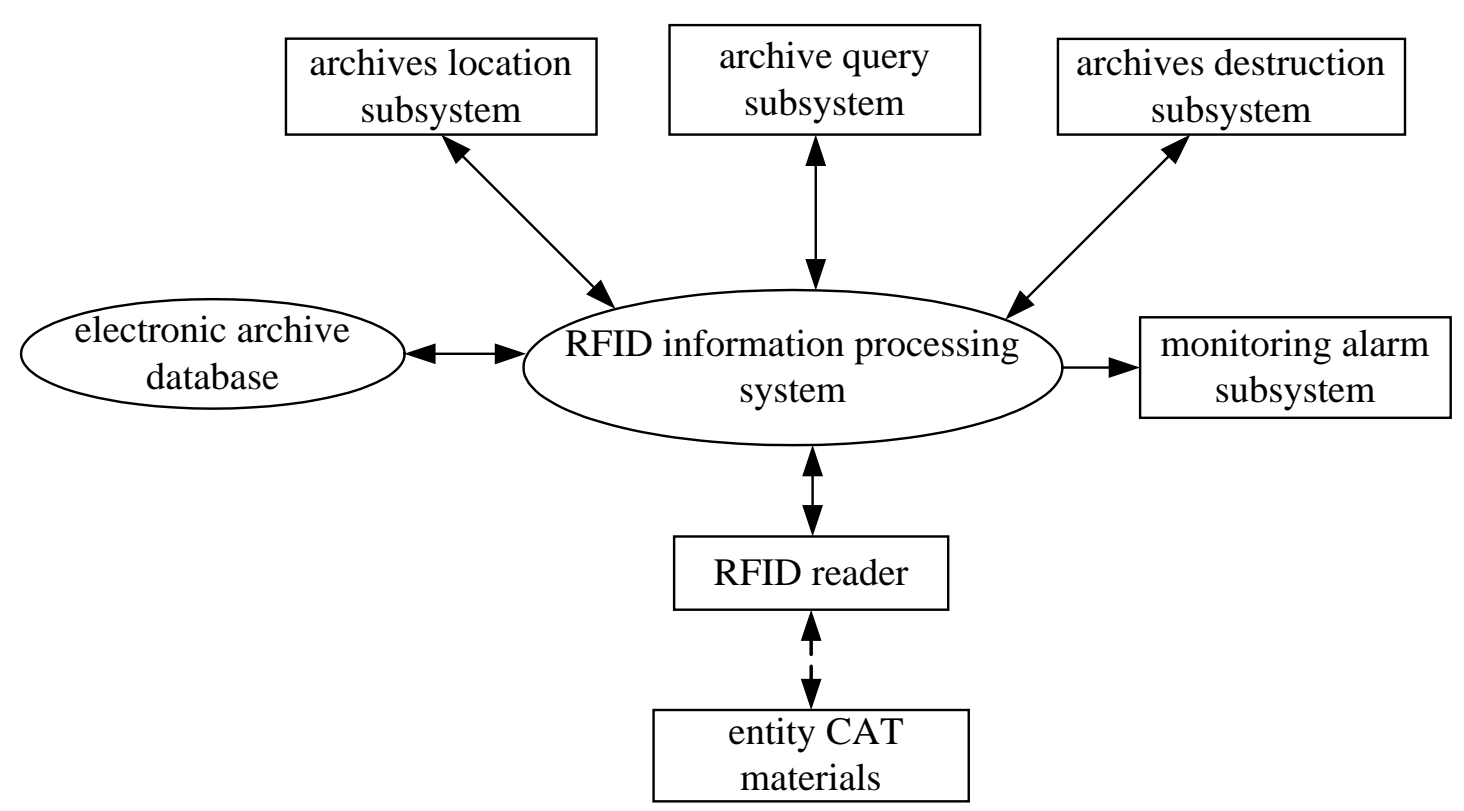

Fig.1 College teaching archive management system based on RFID

Electronic Archive Database. The electronic archive database is run on the backstage server, and electronic archive files are managed in the form of table in database. The tables of electronic archive files and location files are created respectively as

mysql> CREATE TABLE archive (archive number INT(10), location number INT(10),

-> archive name VARCHAR(20), archive type VARCHAR(20),

-> archive security class VARCHAR(20), storage time DATE, storage life DATE);

and

mysql> CREATE TABLE location (location number INT(10), location type

VARCHAR(20), $\quad$-> archive number interval INT(10), number of stored archive

INT(10) );

The information stored in the archive tags and location tags is recorded as above data structures.

RFID Subsystem. The RFID subsystem consists of RFID tags, RFID readers and antennas. The RFID tags are passive, and classified into two types, the archive RFID tags and location RFID tags. The archive tags are packaged in the form of card, and glued on the archive boxes, but only one location tag is stalled in every independent compartment of the archive cabinet. The information stored in a non-volatile memory of the RFID tag cannot be lost under the conditon of power-off. When the RFID subsystem receives read command from RFID information processing system, the reader sends a radio-frequency signal to a passive RFID tag, simultaneously the passive RFID tag converts a magnetic field of the incident reader signal into a power source, which is used to transmit the information to the reader. Both of the archive information and the location information are read by the RFID readers though the antennas.

Archive Query Subsystem. The archive query subsystem provides the query function for teachers to find the valuable teaching materials. The archive query subsystem provides web interfaces, by which teachers can access college teaching archive management system based on RFID, and then can retrieve, read, and download the teaching electronic archive files stored in the electronic 
archive database. Note that teachers can perform access operation only after authorization of the archive query subsystem.

Archive Destruction Subsystem. When the storage life of a college teaching archive is over, the record of the archive will be destructed by the professional for managing archives. Namely the teaching archive will be cancelled though the RFID information processing system, the electronic archive files will be deleted from the electronic archive database, the information stored in the location tags will be updated, and the entity archive file materials will be destructed and taken out from the storage room. Note that only the archive destruction subsystem can send destruction command.

Archive Location Subsystem. The archive location subsystem can display the location of teaching archives, make the teaching archives under stored state and keep a record when the location of an archive is changed. At any moment the administrator can master the quantity, location and arrival place of the teaching archives through the archive location subsystem.

Monitoring Alarm Subsystem. When the entity archive files without checkout are taken out from the storage room, RFID information processing system will monitor this case, then the monitoring alarm subsystem will send the alarm information, alarm picture and alarm sound in real time, and prompt the operator to pay attention to the alarm information and confirm it.

RFID Information Processing System. The RFID information processing system is the kernel of college teaching archive management system based on RFID. It has connections with electronic archive database, RFID subsystem, archive query subsystem, archive destruction subsystem, archive location subsystem, and monitoring alarm subsystem. The archive query subsystem, archive destruction subsystem and archive location subsystem can accomplish operating the electronic archive files in electronic archive database and entity archive files in the storage room only after authorization of RFID information processing system is approved. When the entity archive files without checkout are taken out from the storage room, RFID information processing system will monitor this case through the RFID subsystem, and let the monitoring alarm subsystem start alarm operation.

\section{Summary}

Application of RFID technology in the CTA management is studied in the paper, and a RFID application scheme is presented for promoting the automation of the CTA management. We hope to improve the way in which the college teaching archives are traditionally managed.

\section{Acknowledgment}

This work was supported in part by the Fundamental Research Funds for the Central Universities ( 2015SZYQN17).

\section{References}

[1] Information on http://en.wikipedia.org/wiki/Radio-frequency_identification\#cite_note-18.

[2] Information on http://www.wisegeek.com/what-are-the-different-types-of-rfid-tag-antenna.htm

[3] Information on http://www.mysql.com/. 